\title{
Current Status and Hotspot Analysis of Research on Chronic Disease Management in China
}

\author{
Qiao Liu ${ }^{1}$ Junyong Wang ${ }^{2}$ Li Wang $^{3, *}$ \\ 1 Jiangxi University of Traditional Chinese Medicine, Nanchang, Jiangxi, China \\ ${ }^{2}$ Jiangxi University of Traditional Chinese Medicine, Nanchang, Jiangxi, China \\ ${ }^{3}$ Jiangxi University of Traditional Chinese Medicine, Nanchang, Jiangxi, China \\ *Corresponding author.Email:340088814@qq.com.
}

\begin{abstract}
Objective To analyze the current research status and hotspots of chronic disease management in China in the past decade, and provide a theoretical basis for the development of related research in the later period. Methods: CNKI was used as the search database, with "chronic disease management" or "chronic disease management" as the subject terms, and finally 693 documents were retrieved. On this basis, the 427 documents after screening were analyzed quantitatively, and CiteSpace software was used to analyze The included literature conducts keyword emergence analysis, and obtains the research hotspots in the field of chronic disease management. Results: The number of publications on chronic disease management research topics has been increasing year by year. The journal with the most publications is Chinese General Medicine. There are 25 core authors calculated based on Price's law. The research institution with the most publications is Huazhong University of Science and Technology. School of Medicine and Health Management, Tongji Medical College. After running the CiteSpace software, 10 clusters of keywords including chronic disease management, chronic disease, chronic disease management, and self-management are obtained. Conclusion: The research literature on chronic disease management will continue to rise; the number of cross-institutional and cross-regional cooperative publications is relatively small; the "Internet +" community chronic disease management model will be a hot spot for scholars to study; "The concept of prevention and treatment of chronic non-communicable diseases needs the attention of the industry.
\end{abstract}

Keywords: chronic disease management, self-management, health literacy, community, hypertension

\section{INTRODUCTION}

According to statistics, the number of patients with chronic diseases in China is currently about 300 million, and the annual death toll from chronic diseases accounts for more than $85 \%$ of the total deaths, and the burden of diseases caused accounts for more than $70 \%$ of the total burden of diseases ${ }^{[1]}$, so chronic diseases are a serious threat A type of disease affecting the health of Chinese residents has also become a major public health problem affecting the country's economic and social development. However, since 1972, the United States began to pay attention to the health management of chronic diseases, and in just 20 years, the incidence of cardiovascular and cerebrovascular diseases was reduced by $55 \%$, and the incidence of stroke was reduced by $75 \%{ }^{[2]}$. It can be seen that in the face of a series of problems caused by chronic non-communicable diseases such as cerebrovascular diseases, cancer, chronic respiratory diseases, diabetes, etc., taking effective control and management of them is the focus of our attention. Therefore, this article uses bibliometric research methods and the operation of CiteSpace software to analyze the current status and hot trends of Chinese scholars on chronic disease management research through high-frequency keyword word frequency analysis, co-occurrence network graphs, and cluster analysis. Promote chronic disease management research and provide reference.

\section{DATA SOURCES AND METHODS}

\subsection{Data source}

This article uses the CNKI database as the source of the literature search, using the subject search method, the input condition is "topic: chronic disease management" or "topic: chronic disease management" for search, and the search time is set to "unlimited" -2020", the search time 
is August 2020, and the selected source journals are: SCI source journals, EI source journals, core journals, Chinese Social Science Citation Index (CSSCI) and Chinese Science Citation Database (CSCD), and the preliminary search results 693 documents, excluding monographs, abstract compilations, recommendations of this issue, and other documents irrelevant to the topic, finally included 427 valid documents and saved them in Endnote format. Using Endnote X9 to perform a literature review on the included 427 articles, the results obtained are no duplicate articles.

\subsection{Research method}

CiteSpace is a document visualization software, which displays the development process, research status and hot frontiers of a certain discipline or research field through the mining, analysis, measurement and graph drawing of document data ${ }^{[3]}$. This paper will use Excel software to make descriptive statistics on the publication time characteristics of the included literature and the distribution of the literature source publications, and based on the CiteSpace software to draw the emergence word network map of the included literature, analyze the research hotspots and research fields in the field of chronic disease management in my country. trend.

\section{RESEARCH STATUS}

\subsection{Document publication time and distribution characteristics}

The publication year of the literature can reflect the progress of a certain research in a period of time ${ }^{[4]}$. The publication time of its literature can be roughly divided into a period of exploration (2000-2008), a period of rapid growth (2008-2010), and a period of steady growth (2011-present). On the whole, the literature on chronic disease management research in China is still on the rise in recent years.

\subsection{Literature source journal distribution}

A statistical analysis of the included 427 articles reveals that they are distributed among 79 core Chinese journals, The total volume of articles is 267 , accounting for the total amount of articles published. Of $60.2 \%$. Among them, the top three journals with the number of articles are "Chinese General Practice", "Nursing Research" and "Chinese Health Service Management". The number of articles are 84, 22 and 18 respectively, accounting for $19.7 \%$ and $5.2 \%$ And $4.2 \%$.

According to statistics, the research institution with the largest amount of publications is the School of Medicine and Health Management, Tongji Medical College, Huazhong University of Science and Technology. The first publication was in 2008 and the publication amount was 7; followed by the School of
Public Health of Fudan University and Nanjing Traditional Chinese Medicine. The School of Health Economics and Management of the University and the School of Public Administration of Renmin University of China respectively issued six articles in 2010, 2017 and 2016. Judging from the geographical distribution of the top ten institutions in the number of publications, the research institutions that are relatively concerned about chronic disease management research are all higher education institutions in first-tier or quasi-first-tier cities, indicating that in economically developed regions, scholars are sensitive to chronic disease management research The degree is higher.

\subsection{Distribution of publishing agencies}

According to statistics, the research institution with the largest number of publications is the School of Medicine and Health Management, Tongji Medical College, Huazhong University of Science and Technology. The first publication was in 2008 and the number of publications was 7; followed by Fudan University School of Public Health and Nanjing University of Traditional Chinese Medicine Health Economic Management The school and the School of Public Administration of Renmin University of China have published papers in 2010, 2017 and 2016 respectively, and each has issued 6 articles. Judging from the geographical distribution of the top ten institutions in the number of publications, the research institutions that are relatively concerned about chronic disease management research are all higher education institutions in first-tier or quasi-first-tier cities, indicating that in economically developed regions, scholars are sensitive to chronic disease management research The degree is higher.

\subsection{Analysis of core authors}

The number of papers is an important indicator to measure the individual scientific and technological output of scientific researchers, and it is also an important basis for judging the core author group in a field ${ }^{[5]}$. After statistical analysis of the authors' data, there are a total of 399 authors (first authors) among the 427 papers, of which 374 have published only one paper, It accounts for $93.7 \%$ of the total number. Among them, Hong Jiang of the First Affiliated Hospital of Chongqing Medical University published the most papers, reaching 4 papers. Therefore, the core author is determined based on the core author certification formula of Price's law $(\mathrm{N}=0.749 * \mathrm{nmax} 1 / 2)^{[6]}$. After calculating $\mathrm{N} \approx 2$, that is, the author who has published no less than 2 articles in this field is the core author. There are 25 authors who have met the criteria for more than two papers. The five core authors come from major universities or hospitals, have high medical literacy and academic standards, and have their own research fields for the investigation of chronic 
disease management. His research direction involves many fields such as medical education and clinical medicine, mainly focusing on medical and health policies. Among them, the articles published by five core authors have been recognized and cited by scholars in the same research field. For example, the three core papers published by Lanting Lv of Renmin University of China have a total of 168 citations, and each article is cited on average. The number of times is 56 , indicating that the article has a high academic value.

\section{RESEARCH HOTSPOT EVOLUTION}

\subsection{Keyword emergence analysis}

"A group of emerging dynamic concepts and potential research problems" can represent research hotspots in a certain field ${ }^{[7]}$. Run CiteSpace to get the main emerging keywords and their emergence time in the domestic chronic disease management research field from 2000 to 2020.After sorting out and analyzing the emerging keywords, the research process can be divided into two time periods, which are mainly divided into 2007-2013 and 2017-2020.

The first time period is from 2007 to 2013. During this period, the focus of chronic disease management in China is mainly on community health services, and the community is the unit, using health education, health promotion, and regular physical examinations to improve patients with chronic diseases. The level of self-health management. It is not difficult to find that the keywords of hypertension and diabetes, the two typical chronic non-communicable diseases, have high emergent values. Therefore, it is not difficult to know that cardiovascular and cerebrovascular diseases such as diabetes and hypertension have always been the most common diseases that scholars explore in the field of chronic disease management research.

The second time period is from 2017 to 2020 . During this period, domestic scholars will focus on chronic disease management research from the community's disease management of chronic disease patients to selfmanagement of the lifestyle of chronic disease patients. At this stage, most scholars are concerned about improving the health awareness of patients with chronic diseases and improving the daily habits of patients with chronic diseases through the Internet. For example, smart APP can improve the self-management awareness of patients with chronic diseases with the advantages of convenience and portability. This shows that the current domestic chronic disease management research perspective is no longer limited to conventional hospital treatment or community primary health services, but is concerned with the impact of changes in the social environment on the chronic disease management of patients with chronic diseases, thereby further improving patients with chronic diseases. Make a comprehensive plan for daily management.

\subsection{Analysis of main research hotspots}

After the cluster identification generated by CiteSpace, you can choose specific and practical phrase identification, and finally form a key co-occurrence map that more comprehensively reflects the research hotspots in a certain field, which has a certain forward-looking and objectivity ${ }^{[8]}$. Through the main research directions of keyword cluster analysis, the research results obtained are mainly concentrated on 11 thematic clusters, and the results run by CiteSpace are sorted out, and the main research hotspots of chronic disease management research in China in recent years It is classified into the following points: (1) The current status and influencing factors of chronic disease management. The keywords of this cluster include influencing factor analysis, family doctor contracted service, community health service, disease management, etc. The research objects mainly include family doctors, elderly People, hypertensive patients, medical staff, etc., through content analysis, it can be known that age, education, economic level, and lifestyle are all factors that affect the condition of patients with chronic diseases and the development of chronic disease management. (2) Evaluation and measurement indicators of chronic disease self-management behavior. The cluster mainly includes key words such as risk assessment, self-efficacy, influencing factors, and questionnaire surveys. The research points out that it targets chronic disease patients' living habits, selfmanagement status and health literacy level To evaluate the self-management behavior of chronic diseases and other factors, extract questions related to the research topic from policy provisions such as "Health Literacy of Chinese Citizens-Basic Knowledge and Skills (Trial)", form relevant measurement indicators, and organize them into questionnaires. Among them, the frequency of surveys on the health literacy of patients with chronic diseases and the status quo of self-management of patients with hypertension is more frequent, and they have received higher attention. (3) The exploration and construction of chronic disease management model. This cluster includes key words such as process reengineering, chronic disease management mode, family management mode, and continuous care mode. For the establishment of the chronic disease management system, my country initially borrowed from two internationally recognized chronic disease management models: CCM (Chronic Disease Management Model) and ICCC (Innovative Chronic Disease Care Framework) ${ }^{[9]}$. However, related studies have pointed out that the current chronic disease service system in my country is not yet complete, and there are problems such as insufficient policy environment, lack of legislation, incomplete chronic disease management resources, insufficient multisectoral participation and collaboration, and poor chronic 
disease management effects in communities and residents. It will be the focus of restructuring the chronic disease management system ${ }^{[10]}$. Therefore, the abovementioned domestic chronic disease management problems must be considered in order to establish a chronic disease care system suitable for my country's national conditions. (4) Research on community health services and health management. Its clustering includes major keywords such as community medical care, village clinics, health behavior, community health management, and community medicine. The study pointed out that since the medical reform, community health services have become a hot area of health management research. It is the core area of the elderly in my country, that is, the high-risk group of chronic diseases, which needs to vigorously develop health management ${ }^{[8]}$. (5) The current status of chronic disease prevention and health literacy. Its clustering includes major keywords such as healthy behavior, health literacy, TCM nursing, TCM health management, TCM consortium, self-efficacy, and disease prevention. Relevant studies have pointed out that the management of patients with chronic diseases in the way of traditional Chinese medicine conditioning and the concept of disease prevention is conducive to improving patients' awareness of their own conditions, improving patient compliance, improving patients' clinical symptoms, and improving their quality of life ${ }^{[11]}$. Treating chronic non-communicable diseases with traditional Chinese medicine will become a hot spot that cannot be ignored. (6) The "Internet + " chronic disease management model. Its clustering includes major keywords such as big data, mobile medical care, mobile apps, Internet + and quality of life. According to a study by the National Medical Book Alliance, all medical and health decisions made by individuals are based on the health information obtained [12], and various online medical communities as one of the open windows of "Internet + medical" are just It provides a platform for users to exchange and share health information, and its various services meet the health information needs of users to a certain extent ${ }^{[13]}$. Therefore, the health management of patients with chronic diseases based on the Internet has become a hot spot for researchers and researchers.

\section{CONCLUSION}

The CNKI database is searched on the subject of "chronic disease management" or "chronic disease management", and relevant documents are obtained. Bibliometrics methods are used to analyze the current situation of publication time, source journals, publishing organizations, and core author distribution. And use CiteSpace software to carry out the hot spot analysis of the included 427 core journal articles, and draw the following conclusions.

(1) At present, community health service agencies are still the main mode of chronic disease control recognized and recommended by $\mathrm{WHO}^{[14]}$. Studies have pointed out that the community is the most suitable place to carry out interventions on chronic disease risk factors, which can achieve the greatest benefit to the population, the most cost-effective, and the most effective inhibition of new patients ${ }^{[15]}$. Therefore, most scholars at present are still focusing on the research of chronic disease management on how to improve primary community health services, so as to improve the condition of chronic disease patients and improve their quality of life. However, the establishment of a chronic disease management framework based on the community, scholars have found a uniform problem, such as the medical conditions of the basic-level community health service institutions cannot meet the needs of chronic disease patients, and the lack of a unified service network and health technicians, The medical insurance system is not yet mature and other defects ${ }^{[16]}$. These problems are still the problems of the community chronic disease management model that need to be solved urgently in our country, and breakthrough progress and research have not been made.

(2) According to surveys, $80 \%$ of the elderly suffer from at least one chronic disease, and $50 \%$ of the elderly suffer from two chronic diseases [17], among which hypertension, diabetes, and coronary heart disease are particularly prominent ${ }^{[18]}$. Therefore, most scholars in the field of chronic disease management research in China still focus on common and frequent chronic noncommunicable diseases such as hypertension and diabetes. However, there is no unified and reasonable community management model for chronic diseases such as hypertension in China, and it is still in the discussion stage ${ }^{[19]}$. Therefore, the exploration of the community management model for multiple chronic noncommunicable diseases such as hypertension and diabetes will continue to be a hot spot for Chinese scholars to continue to pay attention and explore.

(3) At present, most of the literature is based on the perspective of patients with chronic diseases as consumers of health care services, to explore how to improve their health literacy level. This allows patients to ask for needs and passively receive health education and health information, often with poor results. Emphasis should be placed on letting patients with chronic diseases as the main providers of health care services to actively learn related knowledge of chronic disease prevention and health management. This will not only improve patients' health literacy and independent management capabilities, but also improve community health services. Efficiency, reducing medical costs and other effects.

(4) With the serious aging of the Chinese population, the elderly, as the main group of patients with chronic diseases, have an increasing demand for health maintenance and management. However, the traditional medical service model cannot meet their needs. In recent years, the "preventive treatment" theory of traditional 
Chinese medicine has provided scientific theoretical support and working methods for preventive health care and health services for the elderly ${ }^{[20]}$, and its advantages in the formulation of coping strategies and health management have gradually emerged. Therefore, the way of Chinese medicine nursing care has attracted more and more attention from scholars in the field of chronic disease management. However, the current Chinese medicine thought has not yet been deeply rooted in the hearts of the people, the relevant propaganda efforts are not strong, the general practitioners are relatively scarce, and there are few related support policies issued [21]. Therefore, the use of traditional Chinese medicine ideas and methods to treat patients with chronic diseases is still a key concern for scholars in the field of chronic disease management research, and it will also be a major trend in future research and development.

In recent years, China has actively promoted the "Internet + medical" health service system. In 2016, the "Guiding Opinions of the State Council on Promoting and Regulating the Application and Development of Health and Medical Big Data" pointed out that it is necessary to conform to the development trend of information technology, standardize and promote the integration, sharing and open application of health and medical big data ${ }^{[22]}$. Big data has brought convenience to community health services. More and more medical wearable devices, mobile APP and other technological devices can monitor patients' health information in real time. However, the rapid expansion of the Internet will also cause many problems, such as difficulty in ensuring patient information security, no specific legal protection framework has been formed, the lack of a corresponding chronic disease data sharing platform, and the formation of "information islands" [23]. Therefore, in the future research, the construction of the "Internet + chronic disease management" model will be a hot research field, but it is also full of difficulties and needs to be improved and considered in many aspects.

\section{ACKNOWLEDGMENTS}

A project funded by the National Natural Science Foundation of China, the evaluation and optimization of chronic disease classification diagnosis and treatment model based on clinical pathways: taking diabetes as an example. (No.71764012)

\section{REFERENCES}

[1] Ye Wang.Interpretation of the main points of the 2012-2015 "China's Chronic Disease Prevention and Control Work Plan"[J].Chinese Journal of Cardiovascular Diseases,2012,40(10):887888.DOI:103760/cma.j.issn.0253-

3785.2012.10.019.
[2] Chao Feng,Qinqin Wu,Jihong Ma,etc.Chronic Disease Management in Health Management Center[J].Modern Preventive Medicine,2008(08):1517-1518.

[3] Ze Li,Songlin Wang,Jing Zhao,etc.Analysis of research hotspots and trends of Chinese family doctor contracted services based on CiteSpace[J]. Chinese General Practice ,2019,22(22):2675-2680.

[4] Yan Zhou.A Statistical Analysis of Research Papers on Public Archives in China from 1999 to 2008[J].History of Heilongjiang,2010,(1):113-116.

[5] Guang Yu,Li Fang.Analysis of authors of papers in "Chinese Journal of Occupational Diseases" from 2006 to 2013[J].Chinese Journal of Occupational Diseases,2014,32(12):903-906.

[6] Yun Xu.Quantitative Analysis of Chinese Medical Papers Based on Chinese Science Citation Database[J].Chinese Journal of Information on Traditional Chinese Medicine,2017,24(05):95-98.

[7] Chen C.CiteSpace II:Detecting and visualizing emerging trends and transient patterns in scientific literature $[\mathrm{J}]$.Journal of the American Society for Information Science and Technology,2006,57(3):359-377.

[8] Zehao Zhang,Xiuyan Chen,Feng Xu,etc.Visual analysis of research on health management discipline based on knowledge graph[J].Chinese Health Service Management,2017,34(07):485$487+497$.

[9] Lanting Lv.The Enlightenment of International Chronic Disease Management Theory Model to China[J].Chinese Journal of Health Information Management,2015,12(05):529-534.

[10] Yuan Yao,Gaoling Wang.Reconstruction of chronic disease management system from the perspective of social management[J].Chinese Health Service Management,2017,34(02):157-160.

[11] Yinji Xu,Yiyan Luo,Lei Wu,etc.The influence of strengthening the management of chronic diseases of traditional Chinese medicine on the knowledge, belief and behavior of patients with chronic obstructive pulmonary[J].Lishizhen Traditional Chinese Medicine,2016,27(01):246-248.

[12] TU H T,HARGRAVES J L.Seeking health care information:most consumers still on the sidelines[J].Issue brief (Center for Studying Health System Change),2003(61).

[13] Huixiang Xiong,Qinquan Xiong,Xiao Mei.Construction of chronic disease knowledge service model for online medical 
community[J].Information studies: Theory \& Application,2020,43(06):123-130.

[14] Jungang Yang,Mingxia Wang,Dehui Dai,etc.Discussion on the Management of Chronic Diseases in the "Four Set Service" Model of Community Health Work[J].Chongqing Medicine,2011,40(30):3110-3111.

[15] Lili Tao,Kaihong Chen,Xiaoyan Han.Analysis of current situation and countermeasures of chronic disease management in community health service stations in a certain district of Beijing[J]. Chinese General Practice,2011,14(34):3959-3961.

[16] Hua Zhang,Xinwei Li,Lingzhong Xu.Community comprehensive prevention and treatment of chronic non-communicable diseases and medical insurance[J].Chinese Health Service Management,2009,26(06):384-386.

[17] Kanasi E, Ayilavarapu S, Jones J.The aging population:demographics and the biology of aging[J].Periodontology, 2016;72 (1) :13-8.

[18] Tey NP, Lai SL, Teh JK.The debilitating effects of chronic diseases among the oldest old in China[J].Maturitas, 2016;94:39-45.

[19] Chongkai Xu,Jianlong Ji,Jing Cao.Exploration of the management model of hypertension with community health service center as the main body[J]. Chinese General Practice,2010,13(01):86-88.

[20] Huiwen Wu,Lin Wang,Zhengning Yang,etc.Analysis of TCM health management needs and talent training in the context of general health[J].Chinese Medicine Bulletin,2020,19(01):38-40+46.

[21] Jing Wang, Yang Yu,Haili Zhang,etc.Discussion on the chronic disease management model of family doctors in conjoined traditional Chinese medicine $[\mathrm{J}]$.Chinese Hospital Management,2020,40(07):94-96.

[22] Tianyu Ye,Gaoling Wang.Research on the Application of Medical and Health Big Data in the Management of Chronic Diseases[J].Health Economics Research,2017(02):67-69.

[23] Ting He,Xing Liu,Ying Li,etc.Research progress in the application of big data analysis in chronic disease management[J].Chinese Public Health,2016,32(07):981-984. 